\title{
BIBLIOTECAS, FOMENTO DE LA LECTURA Y REDES SOCIALES: CONVIRTAMOS AMIGOS EN LECTORES
}

Ramón-Alberto Manso-Rodríguez

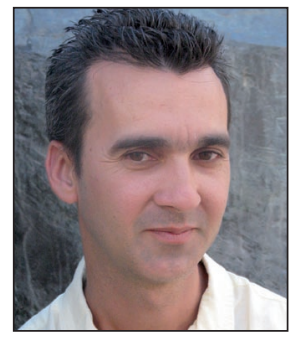

Ramón-Alberto Manso-Rodríguez es doctor en documentación e información científica por la Universidad de Granada, 2010. Desarrolla su actividad como profesor asistente en la Facultad de Ciencias de la Información y la Educación, en la Universidad Central "Marta Abreu" de Las Villas (Cuba) y es miembro del grupo Investigación y Desarrollo de la Biblioteca Pública Provincial. Es becario de investigación en la Universidad de Granada y miembro colaborador del grupo Infosfera.

\author{
Fac. de Ciencias de la Información y la Educación \\ Universidad Central de Las Villas \\ Carret. Camajuaní, Km 5 1⁄2, Santa Clara, Villa Clara, Cuba \\ manso@uclv.edu.cu
}

\section{Resumen}

Se analiza la incidencia de las redes sociales en el desarrollo de los clubs de lectura como servicio para el fomento de la lectura. Mediante un análisis documental se presentan los puntos de vista de varios autores y se examina la presencia de los clubs de lectura en la red social Facebook. Se mencionan sus características, se presentan algunos ejemplos de este tipo de grupos, y se enumeran algunas ideas y procedimientos para configurar esta modalidad de servicio por parte de la biblioteca.

\section{Palabras clave}

Clubs de lectura, Redes sociales, Promoción de la lectura, Bibliotecas, Social media, Clubs de lectura online.

Title: Libraries, the promotion of reading and social networks: converting friends into readers

\section{Abstract}

The article focuses on social networks and their impact on the development of book clubs as a service to the promotion of reading. The views of several authors are presented, based on a document analysis, and the presence of book clubs in the social network Facebook is examined, listing their characteristics and some examples of such groups. Some ideas are offered for procedures libraries can follow to configure this type of services.

\section{Keywords}

Book club, Reading club, Social networking, Reading promotion, Libraries, Social media, Online book clubs.

Manso-Rodríguez, Ramón-Alberto. "Bibliotecas, fomento de la lectura y redes sociales: convirtamos amigos en lectores". El profesional de la información, 2012, julio-agosto, v. 21, n. 4, pp. 401-405.

http://dx.doi.org/10.3145/epi.2012.jul.12

\section{Introducción}

La web 2.0 ha generado un alto grado de interconectividad entre los ciudadanos, brindándoles mayores facilidades para intercambiar archivos textuales, sonoros y visuales, enlaces e imágenes, además de poder compartir gustos y aficiones con su comunidad de amigos, familiares y contactos profesionales. Facebook, Twitter, Googlet, LinkedIn y otros sitios de redes sociales son cada vez más utilizados por una amplia comunidad de usuarios.

Por el contrario, estos usuarios demandan los servicios que las bibliotecas ofrecen en su espacio físico cada día con menor frecuencia. Para revertir esta situación y mantener el contacto social con ellos (Bradley, 2011), los bibliotecarios deberían implicarse en las redes sociales de una forma más activa que el común de los usuarios. Tienen que mostrar a los usuarios cuán útil e importante puede resultar el mantener el contacto con ellos.

Las redes sociales son una plataforma que permite a las bibliotecas una comunicación rápida, eficaz y directa con su comunidad usuaria, además de favorecer la difusión de sus eventos, actividades y materiales documentales (Marquina, 2010).

Bradley (2011) reconoce cinco aspectos que benefician e inciden en un uso diferente de los medios sociales por los bibliotecarios: 
- son un recurso para la búsqueda;

- pueden actuar como filtros en la calidad de la información que se referencia;

- permiten compartir información, produciéndose "inteligencia colectiva";

- permiten influir en la comunidad;

- ciertas búsquedas de información pasan de hacerse de forma individual a social.

Bradley (2011) afirma que mientras más contactos, amigos, links, tweets, fotografías, "me gusta" y +1 consigan los bibliotecarios, mayor influencia se tendrá, lo que provocará en la comunidad un mayor interés por lo que biblioteca está realizando y de esta forma se será más útil a la comunidad usuaria.

En la biblioteca actual, una vía para aprovechar la influencia que las tecnologías 2.0 proporcionan en la formación del conocimiento colectivo, es el fomento y promoción de la lectura. Celaya (2007) dice que las redes sociales son herramientas que ayudan a fomentar una conversación en red entre lectores de libros, actuando como un punto de información y de encuentro participativos, donde se anima el intercambio de opiniones sobre libros y autores.

El mismo autor indica que las "tecnologías sociales permiten a los lectores expresarse libremente sin ningún tipo de intermediación, lo que los convierte en medios idóneos para obtener información y compartir opiniones sobre un determinado libro y/o autor". De esta forma, estos medios complementan la labor de fomento de la lectura que se realiza en las bibliotecas.

Muchas bibliotecas desconocen por dónde empezar, cómo evaluar la eficacia de las redes y de qué forma fomentan la lectura de libros. Este estudio se propone un acercamiento a este tema, primero revisando la bibliografía y posteriormente particularizando en los clubs de lectura virtuales, fundamentalmente en Facebook, para finalmente llegar a conclusiones que permitan valorar entre los bibliotecarios la posibilidad de llevar a cabo esta actividad en ese sitio web.

Las redes sociales son eficaces para fomentar los libros y la lectura

Finalmente el estudio pretende demostrar que es tiempo de que las bibliotecas profundicen en sus proyectos sociales. Como plantea Marquina (2011), los bibliotecarios deben estar presentes donde estén sus usuarios y no estar por estar, sino por convicción de su necesidad.

\section{¿Son las redes sociales una vía para el fomento de la lectura? Análisis de la literatura}

Celaya (2009) se pregunta por qué no se utilizan las redes sociales para atraer la curiosidad de los jóvenes hacia el contenido de los libros y fomentar así el placer de leer, o por qué no se fomentan conversaciones sobre libros en blogs y redes sociales, si es conocido que hoy en día los jóvenes escriben y leen más que nunca en blogs, wikis y redes sociales, siendo capaces además de llevar a cabo varias tareas de forma casi simultánea, a través de múltiples medios digitales.
Según el mismo autor, mediante el empleo de las redes sociales las bibliotecas pueden crear espacios de lectura y escritura más cercanos a la manera en que sus usuarios se comunican actualmente, redundando en una mayor motivación por el placer de leer. Sugiere en su artículo como posibles vías la colaboración de las bibliotecas en la Wikipedia, la grabación y subida a YouTube de vídeos con las actividades realizadas, y la participación en Twitter.

Por su parte Seoane (2008) cita como ventajas de un club de lectura online: potenciar el uso de las colecciones, animar a la lectura, alfabetización informacional, ofrecer información sobre novedades bibliográficas y presentar actividades relacionadas con el libro. La misma autora indica que esta modalidad de club de lectura elimina los límites geográficos y temporales y promueve el debate. Sugiere que debe ser un apoyo al club existente de manera tradicional, donde sus miembros puedan compartir los comentarios y reflexiones en torno a una obra determinada y sirva como su canal de comunicación. Finalmente recomienda que las bibliotecas estén donde están sus usuarios.

El empleo de las redes sociales para la creación de clubs de lectura es considerado por James (2003) como una vía para mejorar la lectura, la escritura, el uso de la tecnología y las habilidades de pensamiento crítico. En su artículo (que presenta una experiencia práctica con estudiantes de primaria), la autora escribe que los clubs virtuales potencian la lectura compartida y favorecen el desarrollo de habilidades sociales gracias a la interacción con estudiantes de otras escuelas. Además, éstos aprenden a usar la tecnología, mejoran sus habilidades con el lenguaje y les facilita la construcción de sus propias bibliotecas personales.

Según Lozano (2010) las bibliotecas tienen la responsabilidad de mantener una política de difusión y promoción de los libros, apostando para ello por la innovación basada en la cooperación, la búsqueda de sinergias y el uso eficiente de los recursos, para ofrecer así actividades con valor añadido de proyección social. Insiste esta autora en la vinculación del fomento de la lectura a la innovación a partir de la presentación de propuestas diferentes, diversificadas, atractivas e impregnadas de la filosofía 2.0, mediante la utilización de herramientas que mejoran la colaboración con los usuarios y otras instituciones. Indica además, que en la actualidad las bibliotecas no pueden desarrollar actividades de animación de la lectura a espaldas de la opinión y necesidades de sus usuarios.

Viendo que fuera del ámbito de las bibliotecas los clubs de lectura estaban prosperando, AuYeung, Dalton y Gornall (2007) Ilevaron a cabo una iniciativa en la Toronto Public Library, que es un referente en esta modalidad de servicio en entornos virtuales. En su artículo enumeran una serie de elementos a considerar para la implementación de un club de lectura virtual, normas y las reglas a observar por los bibliotecarios moderadores, con los pros y los contras.

Otros autores, como De-la-Cruz-González-Cutre y SaurinParra (2008), señalan también la eficacia y rentabilidad de los clubs de lectura online. En su artículo estudian los implementados por las bibliotecas españolas, esbozan sus principales deficiencias, y proponen medidas para que funcionen de manera más eficiente. 
En resumen, son múltiples las posibilidades para implantar servicios virtuales que potencien el fomento de la lectura entre los usuarios. En su gran mayoría los proyectos presentados usan blogs y en menor medida redes sociales como Facebook, Twitter u otras. Las bibliotecas deben considerar ambas alternativas para animar a los usuarios a desempeñar un papel activo, a compartir información, y opinar o valorar los contenidos que les son más interesantes.

El empleo de las redes sociales en el fomento de la lectura ofrece a la biblioteca la posibilidad de mantener un flujo de comunicación constante con los usuarios y éstos a su vez con otros usuarios, beneficiándose ambas partes. Conociendo las recomendaciones de libros entre los lectores, la biblioteca podrá monitorear sus gustos, necesidades e intereses.

El simple hecho de estar presente donde están sus usuarios, en las redes sociales, ofrece a la biblioteca mayor visibilidad, permitiéndole tener un canal de comunicación barato y atractivo, donde poder promocionar las colecciones, servicios y actividades que lleva a cabo y con ello lograr uno de sus objetivos primordiales: el fomento de la lectura.

\section{Clubs de lectura virtuales en Facebook}

Por club de lectura se entiende un grupo informal de lectores que se reúnen para leer un mismo libro y posteriormente comentar y discutir sus puntos de vista entre todos (Reitz, 2010).

Esta actividad la llevan a cabo tradicionalmente casi todas las bibliotecas, pero cada vez menos personas tienen el tiempo necesario para asistir regularmente, por lo que están siendo sustituidos por los clubs de lectura virtuales.

Al adentrarnos en el objeto de estudio, la presencia de los clubs de lectura en la red social Facebook y su posterior análisis, se hizo necesario normalizar los datos a recolectar sobre cada página. En este sentido se tomaron como elementos: título del club, dirección web, objetivos o público al que va dirigido, organización que lo desarrolló, tipo de información que referencia en su muro, servicios adicionales que ofrece y cantidad de usuarios.

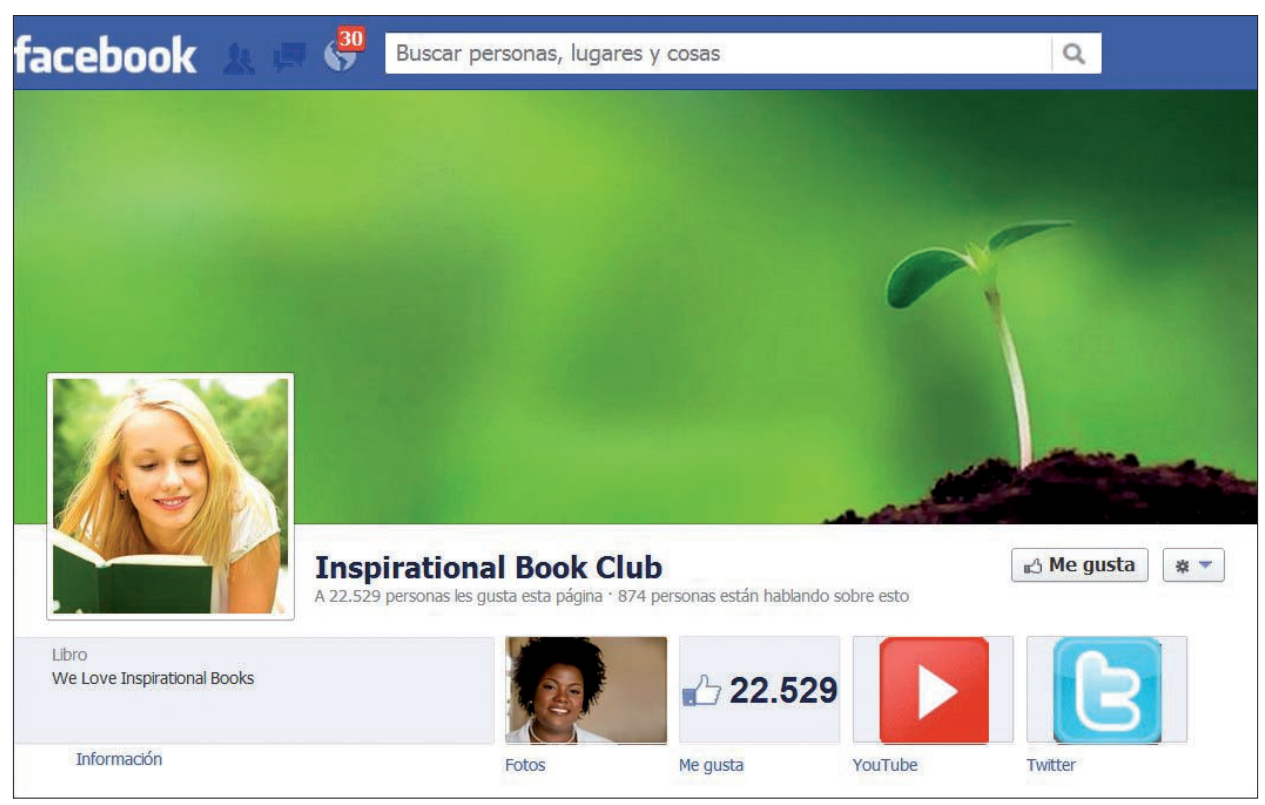

La decisión de investigar en Facebook viene dada por su popularidad, así como por su facilidad de uso y potencialidades para organizar contactos, crear grupos, compartir fotografías, insertar textos con enlaces, interactuar con la comunidad y desarrollar aplicaciones. Los clubs más notorios, tanto en el ámbito anglosajón como hispano, son:

\section{Science Fiction Book Club}

\section{http://www.facebook.com/ScienceFictionBookClub}

Informa sobre libros de ciencia ficción y fantasía. Tiene 35.135 usuarios y en su muro ofrece la descripción de los libros, incluyendo la portada. Permite compartir los recursos, comentarlos y cuenta con la opción "Me gusta".

\section{Book Club}

\section{http://www.facebook.com/bookclubbooks}

Ofrece sugerencias sobre libros con sus portadas y comentarios respectivos. Incluye una sección de "Notas" con datos de autores y publicita los libros en la sección "Eventos". Posee las opciones básicas de "Me gusta", "Compartir" y "Comentar", indicando además en la información del perfil que si el usuario no encuentra un libro determinado se sugiera en el muro. Esta página es seguida por 23.304 personas.

\section{Inspirational Book Club}

\section{http://www.facebook.com/InspirationalBookClub}

Página personal, seguida por 20.915 usuarios, dedicada a ayudar a los interesados en temas espirituales. Acompaña los datos de los libros con foto de la portada y citas a textos del mismo. Permite compartir recursos, comentarlos y la opción "Me gusta”.

\section{History Book Club}

\section{http://www.facebook.com/HistoryBookClub}

Página comercial que promueve libros sobre historia. Tiene 8.409 seguidores. En el muro anuncia los libros de la entidad, incluyendo portada y comentario. Además de las opciones básicas de "Me gusta", "Compartir" y "Comentar", permite que otras personas publiquen en el muro.

\section{Lecturalia}

http://www.facebook.com/ Lecturalia\#!/Lecturalia

Es la mayor comunidad de lectores en castellano, seguida por 36.499 personas. En su muro publica recomendaciones de libros, reseñas y opiniones. Incluye las opciones "Compartir", "Me gusta" y "Comentar", permitiendo de igual forma que los usuarios publiquen notificaciones sobre actividades de su interés.

\section{Quelibroleo.com}

http://www.facebook.com/ quelibroleopuntocom

Este grupo se promociona como el espacio para encon- 


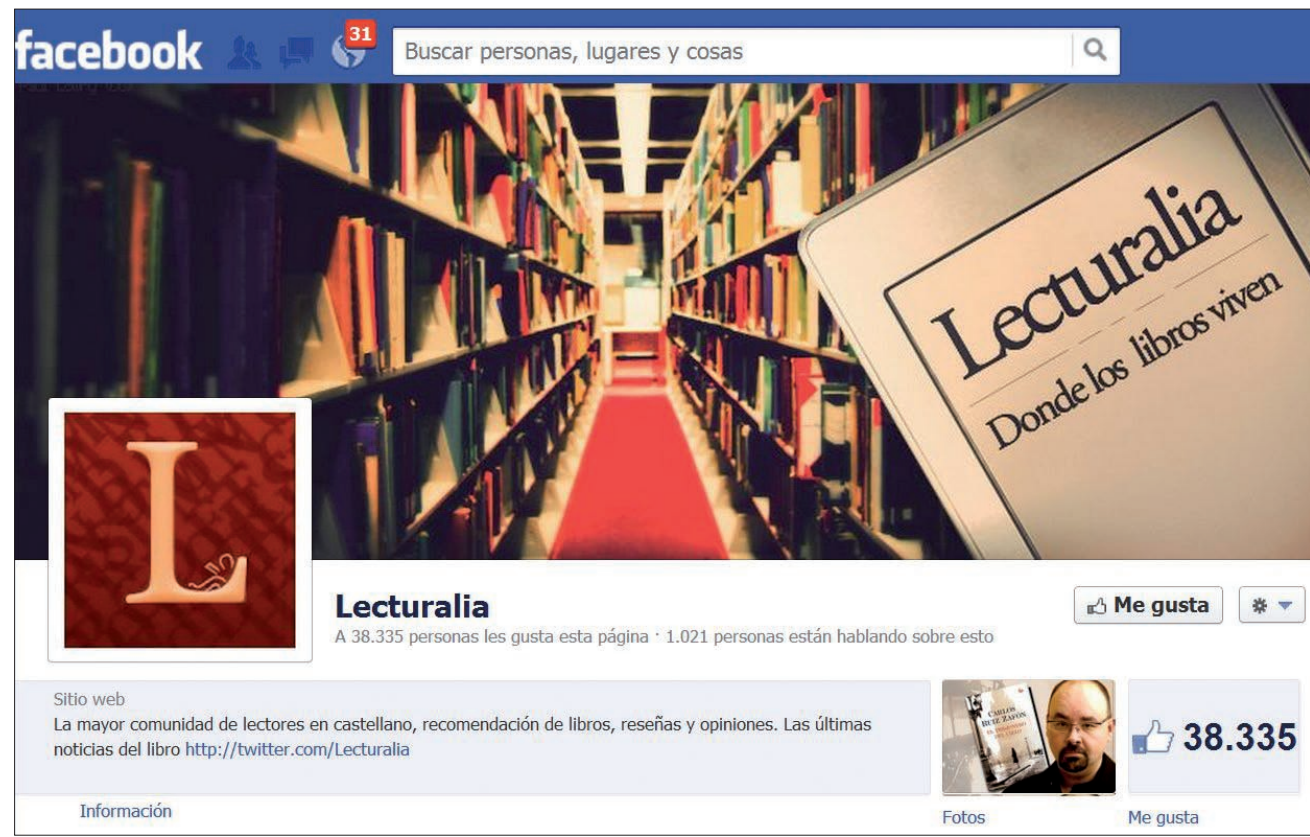

Pública de Albacete, está especializado en literatura de género: novela negra, aventuras, fantasía, terror, policíaca y ciencia ficción. Sólo es seguido por 330 usuarios. En el se promocionan las actividades del club, se recomiendan libros y se publican otras notificaciones de interés. Posee las opciones básicas de "Compartir", "Me gusta" y "Comentar" y la de publicar notificaciones en el muro de la misma.

De los 10 perfiles analizados, sólo uno ha sido creado por una biblioteca; el resto son sitios comerciales o creados por intereses

trar la lectura que recomiendan otros lectores con gustos afines. Cuenta con 25.040 miembros. Posee diferentes secciones: Eventos, Noticias, Youtube, Usuarios activos y Recomendaciones de libros. Tiene otra página asociada bajo el perfil Club de lectura Quelibroleo.com. En su muro propone la lectura de 6 libros y por votación de los usuarios se elige el libro que se leerá en un período determinado. Se pueden hacer comentarios y publicar notificaciones.

\section{Entrelectores}

\section{http://www.facebook.com/entrelectores}

Este grupo es seguido por 12.287 personas. Además de la promoción de lectura mediante la publicación de la imagen de la portada y reseña del libro, se pueden encontrar comentarios e imágenes sobre el mundo de los libros y las bibliotecas. Posee las opciones básicas de "Compartir", "Me gusta" y "Comentar" y los usuarios pueden publicar notificaciones en el muro.

\section{Libros.com}

\section{http://www.facebook.com/libroscom}

Es seguida por 3.085 personas. Publica notificaciones de los usuarios, sus eventos, y promociona los libros permitiendo valorarlos.

\section{Club de Lectura El bolígrafo de gel verde}

\section{http://www.facebook.com/groups/240505485973080}

Es el primer club de lectura organizado en España, en torno a una novela, a través de Facebook. Es una página personal creada por el propio autor de la novela y en ella se puede charlar y comentar sobre la misma. Es un grupo seguido por 1.601 miembros.

\section{Club de Lectura El grito}

http://www.facebook.com/pages/Club-de-Lectura-ElGrito/142176450847

Organizado por la Asociación Cultural El Grito y la Biblioteca personales de un usuario. Todos tienen en común que son espacios colectivos para descubrir un nuevo escritor, conversar sobre determinada obra, intercambiar comentarios o recomendar la lectura de un libro.

Si bien estos clubs se han planteado como objetivo primordial el de promover y mejorar la lectura y algunos logran una considerable cifra de usuarios, lo cierto es que en todos los casos se nota un muy reducido número de comentarios de los lectores en las obras recomendadas. También resalta como elemento común la presencia de enlaces o informaciones relacionadas con las actividades del club, o sobre temas del libro y la lectura.

\section{De los 10 sitios analizados, sólo 1 ha sido} elaborado desde una biblioteca, el resto son sitios comerciales o personales

\section{Perfil del club de lectura de la biblioteca en Facebook}

De la observación realizada, un elemento a considerar es que si la biblioteca organiza sistemáticamente clubs de lectura en su forma tradicional, ¿por qué no se hace también en las redes sociales como Facebook, si muchas bibliotecas tienen un perfil creado en dicha red?

Se necesita un moderador que conduzca el debate, y su desarrollo online difiere poco del tradicional en cuanto a las tareas que se han de llevar a cabo. En este sentido, los pasos a realizar serían:

- crear la página o grupo en la red social; tratándose de un servicio más participativo que informativo es mejor crear un grupo que funcionará como un punto de encuentro. Los miembros, reunidos en torno a un tema común, se conocen, comparten y establecer nuevas relaciones. En Facebook los grupos facilitan la participación más que la páginas. 
- definir las categorías de usuarios a las que se dirigirá;

- determinar los temas a tratar;

- establecer las normas, reglas y políticas del servicio;

- publicitar y difundir el grupo, invitando a los usuarios potenciales a unirse al mismo;

- crear los contenidos para la página: digitalizar las portadas de los libros y ofrecer información detallada de las obras, incluyendo datos del autor, sobre todo si no es muy conocido;

- proponer títulos de libros para que mediante un sistema de votación los miembros decidan qué van a leer;

- guiar el debate entre los lectores;

- supervisar a los usuarios infractores de la política del servicio, y alertar ante incidencias;

- promocionar las actividades del club tradicional y otras actividades de la biblioteca;

- tramitar las sugerencias de los usuarios en cuanto a propuestas de títulos a adquirir.

Otras consideraciones estarían en el orden de asesorar en la selección de un libro o cómo participar de forma activa en el grupo online; también se podría invitar a autores y ofrecer la oportunidad a los usuarios de que se comuniquen con él. Las reseñas de los libros y los comentarios de los bibliotecarios deben ser redactados en estilo coloquial, para facilitar la comunicación. También podría ser un motor impulsor para atraer nuevos usuarios la convocatoria de concursos relacionados con la lectura, como la realización de reseñas de un libro o tema especifico; de esta forma se contribuye al desarrollo de habilidades de comunicación en la comunidad usuaria.

\section{Conclusiones}

El empleo de las redes sociales es una vía idónea para llevar acciones que potencien el fomento de la lectura entre los usuarios de la biblioteca, además de contribuir al desarrollo de las habilidades de información y comunicación en la comunidad, propiciar su participación activa y estimular el intercambio de contenidos.

Las redes sociales, particularmente Facebook, están siendo ampliamente empleadas por otras instituciones y por particulares para promocionar y recomendar libros. Por tanto, es necesario que las bibliotecas avancen en este sentido y, considerando las expectativas de su comunidad usuaria, también aprovechen esta oportunidad para satisfacer sus necesidades formativas e informativas.

Implementar en el espacio virtual los clubs de lectura con la ayuda de las redes sociales, especialmente de Facebook, es una realidad que las bibliotecas tienen hoy al alcance de la mano. Se trata de ver la intervención del usuario como una oportunidad y no como una amenaza, de manera que se potencien también los servicios tradicionales, convirtiéndolos en innovadores. Las opiniones, evaluaciones y recomendaciones serán el valor añadido que contribuya al éxito de la actividad y aumente la visibilidad de la biblioteca.

\section{Referencias}

AuYeung, Catherine; Dalton, Sheila; Gornall, Sandra. "Virtual reading clubs and what we've learned about them". Partnership: the Canadian journal of library and information practice and research, 2007, v. 2, n. 2.

http://www.criticalimprov.com/index.php/perj/article/view Article $/ 237 / 550$

Bradley, Phil. "Which social network should I use as a librarian?" Phil Bradley's website. Making the net easier, 2011. http://www.philb.com/articles/whichsocialnetwork2.htm

Celaya, Javier. "Las redes sociales en el fomento de la lectura en bachillerato". Proyecto de lectura para centros escolares, 2009.

http://www.plec.es/documentos.php?id_seccion=6\&id_document $o=155$ \&nivel=Bachillerato

Celaya, Javier. "Fomento de la lectura en Red". Dosdoce. com, 26 junio 2007.

http://www.dosdoce.com/articulo/opinion/2832/fomentode-la-lectura-en-red

De-la-Cruz-González-Cutre, Inés; Saurin-Parra, Julia. Un viaje virtual por los clubs de lectura, 2008.

http://eprints.rclis.org/bitstream/10760/12561/1/Art._ Blogs-De_la_Cruz\%26Saurin.pdf

González, David. "Cinco redes sociales de libros para convertirte en crítico literario". Lainformacion.com, 18 abril 2012. http://noticias.lainformacion.com/arte-cultura-y-especta culos/literatura/cinco-redes-sociales-de-libros-para-conver tirte-en-critico-literario_QzMQmkXIxoirYaiOxpvW62

James, Jacqueline. "Changing students' attitudes about reading through book clubs". Library media connection, Apr/May 2003, v. 21 n. 7, pp. 30-32.

Lozano, Roser. "El fomento de la lectura en la biblioteca pública 2.0: una apuesta por la innovación y el riesgo". Anuario ThinkEPI, 2010, pp. 87-90.

http://www.thinkepi.net/el-fomento-de-la-lectura-en-labiblioteca-publica-20-una-apuesta-por-la-innovacion-y-elriesgo

Marquina, Julián. "El uso de Twitter y Facebook en las bibliotecas". JuliánMarquina..., 27 sept. 2010.

http://www.julianmarquina.es/uso-twitter-facebook-biblio tecas

Reitz, Joan M. Odlis: Online dictionary for library and information science, 2010.

http://www.abc-clio.com/ODLIS/about.aspx

Seoane-García, Catuxa. A chegada da web social aos clubs de lectura. 2008.

http://www.slideshare.net/catuxa/la-web-social-en-unclub-de-lectura-presentation 